\title{
Correspondence
}

\section{A New Time Delay Estimation in Subbands for Resolving Multiple Specular Reflections}

M. R. Azimi-Sadjadi, S. Charleston, J. Wilbur, and G. J. Dobeck

\begin{abstract}
In this correspondence, a new time delay estimation procedure is proposed using the multiresolution analysis framework through a discrete wavelet transform (DWT). Once the signals are decomposed, the time delays are estimated iteratively in each sub-band using two different adaptation mechanisms that minimize the mean squared error (MSE) between the reference and primary signals in the corresponding sub-band and level. The localization of the minima of the MSE curves at different levels and subbands is used in order to arrive at the time delay estimates. The proposed scheme is then applied to a real-life problem of underwater target detection from the acoustic backscttered data.
\end{abstract}

Index Terms - Adaptive filtering, sonar, time delay estimation, underwater target detection, wavelets.

\section{INTRODUCTION}

In underwater target detection, the presence of a target can be determined by extracting certain clues in the acoustic backscattered signal. However, to extract these clues that are usually dependent on the physical properties of the target and the type of insonification, specular returns must be identified in the backscattered signal [1]. This can only be made possible if the time delays associated with the specular returns can accurately be estimated, especially in the presence of high-level clutter and noise. Depending on the target geometry, beam width, and surrounding environment, the acoustic backscattered signal may contain several closely spaced specular returns. Surface and volume reverberation, competing clutter with similar returns, and other factors such as nonrepeatability of the target signatures and lack of a priori information about the targets can also add to the complexity of this problem.

Time delay estimation (TDE) has been a problem of interest for several decades and has found applications in other areas including radar, speech processing, and biomedical signal processing. The conventional TDE methods such as generalized cross-correlationbased methods [2]-[4] have been shown to have only limited success when applied to real-life problems, especially in the presence of highlevel noise and clutter. Bell and Ewart [5] developed a generalized " $n$-dimensional matched filtering" approach in which the time delays and amplitudes of the paths are determined by maximizing the output of the matched filter. The efficiency and robustness performance of the algorithm in comparison with the standard matched filter was demonstrated on the simulated ocean acoustic data that contained multipath and noise. In [6], a least-squares (LS)-based method for estimating the amplitudes and time delays of multiple reflections in

Manuscript received June 5, 1996; revised May 14, 1998. This work was supported by the Office of Naval Research (ONR 321TS). The Technical Agent was Coastal Systems Station, Panama City, FL. The associate editor coordinating the review of this paper and approving it for publication was Prof. Victor A. N. Barroso.

M. R. Azimi-Sadjadi is with the Department of Electrical Engineering, Colorado State University, Fort Collins, CO 80523 USA.

S. Charleston is with the Department of Electrical Engineering, Universidad Autónoma Metropolitana, México City, México.

J. Wilbur and G. Dobeck are with the Research and Technology Department, Coastal Systems Station, Panama City, FL 42307-5000 USA.

Publisher Item Identifier S 1053-587X(98)08819-9. ocean acoustic backscattered signal is developed. The TDE problem is first converted to the frequency estimation in the frequency domain. The LS method is then applied to provide estimates of the amplitudes associated with each path in the multipath environment. Once the amplitudes are estimated, the time delays are computed using the Gauss-Newton minimization approach. In this TDE method, the number of paths is assumed to be known.

This correspondence presents a new sub-band adaptive filtering scheme that uses DWT and two different adaptation processes for multiple time delay estimation. The estimated delays at different levels/subbands can be validated against each other to provide fairly accurate estimates of the time delays. In addition, as a result of decimation and filtering in the filter bank, signal decorrelation and improved signal-to-noise ratio (SNR) are achieved in each sub-band.

\section{Modelling of Acoustic Backscattered SignAL}

Let us consider the following model for the acoustic backscattered signal, which consists of the target specular returns, multipath reflections, and additive background noise, i.e.,

$$
y(n)=\sum_{i=1}^{M} z_{i}\left(n-\Delta_{i}\right)+e(n)
$$

where

$y(n) \quad$ collected backscattered signal;

$z_{i}\left(n-\Delta_{i}\right)$ ith reflection with time delay $\Delta_{i}$;

$e(n) \quad$ additive ambient noise.

Note that these reflections may or may not be overlapping in time. The above model is arrived at based on the assumption that the target returns and the multipath/reverberation effects have similar signal characteristics. The returns can be modeled as the distorted or modified version of the incident signal, i.e.,

$$
z_{i}\left(n-\Delta_{i}\right)=s_{i}(n) * x\left(n-\Delta_{i}\right)
$$

where

* convolution operation;

$x(n)$ incident signal

$s_{i}(n)$ impulse response of the unknown time-varying system representing the effects of propagating media on the incident signal.

Having modeled the process using (1) and (2), now, in order to identify the specular returns in the backscattered signal, the associated time delays must be accurately estimated. This may be done by using an adaptive transversal filter to undo the effects of the media by approximating the inverse response of the unknown linear system $s_{i}(n)$ and then estimating the time delays. However, since the backscattered signal is quite noisy, DWT can be employed prior to the sub-band adaptive filtering to perform more accurate time delay estimation. This is described in the next section.

\section{Time-Delay Estimation in Subbands}

\section{A. TDE Process}

The proposed TDE process in each sub-band is demonstrated in Fig. 1. The basic principle behind the proposed scheme is that due 


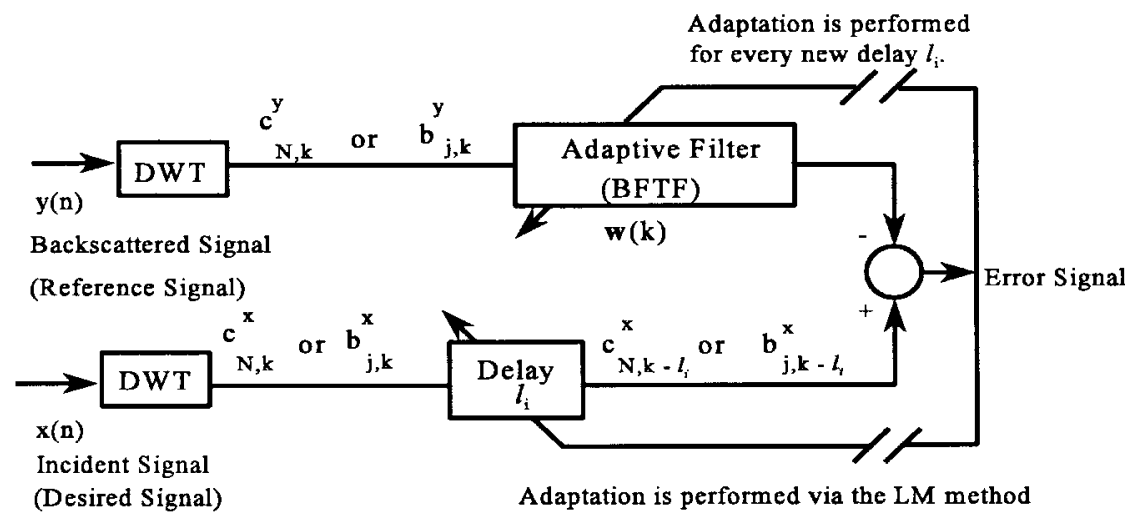

Fig. 1. TDE in subbands.

to the multiresolution property of the DWT, signals in the subbands provide complementary information about the time delays between the reference and desired signals. Consequently, this enables us to gain multiple "looks" of the same signal at different scales/subbands.

Let us assume that the square summable incident signal $x(n), n \in$ $\boldsymbol{Z}$ is decomposed using an iterated filter bank [7], [8] consisting of $N$ levels. If the lowpass and highpass filters in each analysis bank are denoted by $g_{1}(n)$ and $h_{1}(n)$, respectively, then the final lowpass approximation sequence, after subsampling by two in each level, can be written in terms of the impulse response of the halfband lowpass filter as

$$
c_{N, k}=\sum_{n} x(n) g_{N}\left(2^{N} k-n\right)
$$

where $g_{N}(n)$ is

$$
g_{N}(n)=\sum_{k} g_{N-1}(k) g_{1}(n-2 k) .
$$

In a similar way, the "added details" sequences at different level $j \in[1, N]$ can be written in terms of the halfband highpass filters as

$$
b_{j, k}=\sum_{n} x(n) h_{j}\left(2^{j} k-n\right)
$$

where $h_{j}(n)$ is given by

$$
h_{j}(n)=\sum_{k} g_{j-1}(k) h_{1}(n-2 k), \quad j \in[2, N] .
$$

We assume that the filters are of FIR type of order $L$ and the impulse responses $h_{1}(n)$ and $g_{1}(n)$, and their shifted versions by even shift form orthogonal sets [7], [8]. Note that $L$ has to be even in order for the filters to form orthogonal sets. Additionally, we assume that the lowpass and highpass filters are related by the alternating flip, i.e., $h_{1}(n)=(-1)^{n} g_{1}(L-1-n)$ as the modulation by $(-1)^{n}$ indeed transforms a lowpass to a highpass filter [8]. Consequently, the orthogonality of $h_{1}(n)$ and $g_{1}(n)$ with respect to even shifts follows.

The original sequence $x(n)$ can be recovered by upsampling by 2 in each level followed by convolution with filters with impulse responses $\tilde{g}_{1}(n)$ and $\tilde{h}_{1}(n)$ that these are time reversed [7], [8] versions of $g_{1}(n)$ and $h_{1}(n)$, respectively, i.e., $\tilde{h}_{1}(n)=h_{1}(L-1-n)$ and $\tilde{g}_{1}(n)=g_{1}(L-1-n)$. Then, the synthesis equation in terms of these sequences and the corresponding impulse responses of the filters in the associated bands is given by

$$
x(n)=\sum_{j=1}^{N} \sum_{k} b_{j, k} \tilde{h}_{j}\left(n-2^{j} k\right)+\sum_{k} c_{N, k} \tilde{g}_{N}\left(n-2^{N} k\right) .
$$

Note that the above DWT analysis and synthesis equations (3), (5), and (7) are obtained based on the assumption that the signals are of infinite extent. In practice, however, only finite extent signals are encountered, in which case, in order to avoid border problems, we assume that the original signal is symmetrically extended [7], [8].

Once the signals are decomposed, the time delays are estimated in each sub-band iteratively using two adaptive learning mechanisms, as shown in Fig. 1. In this figure, $c_{N, k}^{x}$ and $c_{N, k}^{y}$ represent the final lowpass approximation of $x(n)$ and $y(n)$, respectively, and $b_{j, k}^{x}$ and $b_{j, k}^{y}, j \in[1, N]$ correspond to the added details in different sub-band for $x(n)$ and $y(n)$, respectively. The delay $l_{i}$ is updated to provide an estimate of $\Delta_{i}$, i.e., the time delay associated with the $i$ th return. Note that the final lowpass approximation and the added detail approximations for a delayed sequence $x(n-\Delta)$, with $\Delta=2^{N} l$ and $l$ an integer are, respectively, $c_{N, k-l}^{x}$ and $b_{j, k-2 N-j l}^{x}, j \in[1, N]$. The updating rule for $l_{i}$ is based on the Levenberg-Marquart (LM) algorithm [9]. For every new $l_{i}$, the function of the adaptive transversal filter is to generate an output signal that minimizes the MSE or maximizes the correlation or similarity between the outputs of the delay unit and the adaptive filter. The original block fast transversal filter (BFTF) [10] algorithm is used to update the weights of the adaptive filter. The process is repeated for estimates of the delay $l_{i}$, and the MSE curve is examined for its minima. The positions at which these minima occur correspond to the locations where the maximum correlation between the outputs is found, which in turn correspond to the estimates of the delays $\Delta_{i}$. To account for the time lag introduced by the FIR adaptive filter at these positions, the lag associated with the largest weight is subtracted from the delay estimate in each level. The final delay value is then calculated using $\Delta_{i}=2^{j}\left(l_{i}-q\right)$, where $j$ is the level index, $l_{i}$ is the estimated delay, and $q$ is the lag associated with the weight of the largest magnitude.

The procedure for the selection of the time delays in different levels is based on the following two principles. As a result of the multiresolution decomposition, a) the lower levels (finer scales) offer increased accuracy in comparison with the upper levels (lower scales), and b) the correlation among samples in the upper levels is weaker than those in the lower levels, thus improving the convergence behavior of the LM method as we go down in the decomposition hierarchy. Based on these principles, a decision rule was arrived at in order to select the time delays between two levels. If the number of minima is the same in two consecutive levels, the time delay information is generally extracted from the lower level due to its increased accuracy. The discrepancy in the number of minima is generally caused by high-level noise and clutter in which case, the time-delay information is extracted from the next level due to the improved SNR. A simple thresholding operation of the MSE curve can then result in the estimates of the time delays. It must be pointed out that the minimum delay separation that can be resolved using 
this TDE method is greatly dependent on the incident signal and its length, the decomposition level, and the power of noise and clutter.

It is evident that since the signals at subbands are only periodically time invariant, some inaccuracies in the time delay estimates are inevitable. However, the hierarchical structure of this sub-band TDE method can be exploited to improve the accuracy. That is, once the time delays are estimated at a particular decomposition level, the estimates can be fine-tuned by repeating the process at the previous level only in the vicinity of the detected delays. This process can be continued for several consecutive levels.

\section{B. Adaptation Processes}

Consider, for the sake of simplicity, only the final lowpass approximations for the sequences $x(n)$ and $y(n)$, i.e., $c_{N, k}^{x}$ and $c_{N, k}^{y}$, respectively. The same procedure can also be applied to all the other subbands. In addition, the lowpass approximation $c_{N, k-l_{i}}^{x}$ for the $i$ th specular component will be used as the desired signal for the adaptation mechanisms. In this case, the cost function to be minimized with respect to both the adaptive filter weights and the delay $l_{i}$ is given by

$$
\xi_{N}=\sum_{k}\left(c_{N, k-l_{i}}^{x}-\boldsymbol{w}_{k}^{t} \boldsymbol{c}_{k}^{y}\right)^{2}
$$

where $\boldsymbol{w}_{k}=\left[w_{0}(k) \cdots w_{P-1}(k)\right]^{t}$ and $c_{k}^{y}=\left[c_{N, k}^{y} \cdots c_{N, k-P+1}^{y}\right]^{t}$ are the weight vector for the adaptive filter of order $P$ and its input vector at time $k$, respectively. As mentioned before, $l_{i}$ is updated in each sub-band, using a nonlinear adaptation process via the LM algorithm [9], whereas for every new $l_{i}$, the minimization of (8) with respect to $\boldsymbol{w}_{k}$ is performed using the BFTF algorithm [10].

For the time-delay adaptation, the goal is to find, iteratively, the values of $l_{i}$ that lead to the minimum of the cost function (8). This is done using the adaptation rule as in

$$
l_{i}(t)=l_{i}(t-1)+\Delta l_{i},
$$

where $t$ is the iteration index, and $\Delta l_{i}$ is the updating step. The updating step $\Delta l_{i}$ can be generated using different adaptation approaches [10]. The LM algorithm is a nonlinear LS-based method that provides a mean for interpolating between the Gauss-Newton and the steepestdescent steps. The algorithm possesses quadratic convergence close to a minimum, where it approximates the Gauss-Newton method, whereas, when the initial estimates are relatively poor, it converges like the method of steepest descent [10].

The LM step $\Delta l_{i}$ that minimizes $\xi_{N}$ is given [10] by

$$
\Delta l_{i}=\operatorname{Int}\left[\left(\boldsymbol{J}^{t}\left(l_{i}\right) \boldsymbol{J}\left(l_{i}\right)+\beta\right)^{-1} * g\left(l_{i}\right)\right]
$$

where Int $[x]$ represents the integer part of $x$, and the gradient and the elements of the Jacobian are defined by

$$
\begin{aligned}
g\left(l_{i}\right) & =\boldsymbol{J}^{t}\left(l_{i}\right) \boldsymbol{\epsilon}=2 \sum_{k}\left(c_{N, k-l_{i}}^{x}-\boldsymbol{w}_{k}^{t} c_{k}^{y}\right) \frac{\partial c_{N, k-l_{i}}^{x}}{\partial l_{i}} \\
J_{k}\left(l_{i}\right) & =2 \frac{\partial c_{N, k-l_{i}}^{x}}{\partial l_{i}}=2^{N+1} \sum_{n} x(n) g_{N}^{\prime}\left(2^{N}\left(k-l_{i}\right)-n\right)
\end{aligned}
$$

and $g_{N}^{\prime}(\cdot)$ is computed using the forward difference method [10]. The parameter $\beta$ prevents the possibility of singularity of $\boldsymbol{J}^{t}\left(l_{i}\right) \boldsymbol{J}\left(l_{i}\right)+\beta$. If this parameter is close to zero, then (10) is reduced to that of the Gauss-Newton method, whereas if $\beta$ is greater than $\boldsymbol{J}^{t}\left(l_{i}\right) \boldsymbol{J}\left(l_{i}\right)$, then (10) provides the steepest-descent step. Note that $\epsilon$ represents the error vector with elements $e_{k}:=\left(c_{N, k-l_{i}}^{x}-\boldsymbol{w}_{k}^{t} c_{k}^{y}\right)$. The selection of the parameter $\beta$ in (10) is a crucial step in the application of the LM algorithm. One method for the selection of this parameter is to

\begin{tabular}{|c|c|c|c|c|}
\hline Case & $\operatorname{SNR}(\mathrm{dB})$ & Actual Amplitude & Actual Delays & Estimated Delays \\
\hline 1 & 15 & $1.0 .5,0.4,0.5$ & $0,150,280,420$ & $0,144,288,428$ \\
\hline 2 & 15 & 1.0 .8 .0 .7 .0 .5 & 0.220 .360 .400 & $0.240,368.408$ \\
\hline 3 & 15 & $1,0.2,0.3,0.2$ & $0.150,270,370$ & 0.144 .368 \\
\hline 4 & 15 & $1,0.5 .0 .7$ & $60,300,422$ & $60,300,420$ \\
\hline 5 & 10 & $1,0.5,0.7$ & $60,300.422$ & $60,292,416$ \\
\hline 6 & 2 & $1,0.5 .07$ & 60.300 .422 & $60,308,428$ \\
\hline
\end{tabular}
decide whether the cost function at hand is adequately represented by
TABLE I

ACtual Amplitudes AND Delays FOR the Simulated Backscattered Signals

a quadratic model so that the convergence of the algorithm to a local minimum is guaranteed [10]. The criterion can be written as the ratio

$$
v=\frac{\xi_{N}\left(l_{i}\right)-\xi_{N}\left(l_{i}+\Delta l_{i}\right)}{\boldsymbol{J}^{t}\left(l_{i}\right) \boldsymbol{\epsilon} * \Delta l_{i}+1 / 2\left(\boldsymbol{J}^{t}\left(l_{i}\right) \boldsymbol{J}\left(l_{i}\right)+\beta\right) * \Delta^{2} l_{i}}
$$

where the numerator represents the actual change in the value of the cost function (8), whereas the denominator represents the ideal change provided by a quadratic model of the cost function. A value of $v$ equal to or greater than 1 represents an acceptable model representation, whereas a value less than 1 represents an inadequate model representation. The value of $\beta$ is not modified if $v$ is inside the admissible modeling region, which is between 0.25 and 0.75 [10]. However, outside this interval, the strategy is to select an initial value of $\beta$ and then increase (or decrease) it until the acceptable interval of $v$ is satisfied.

The second adaptive process considered in (8) involves updating of the filter weights using the BFTF algorithm [10]. This algorithm is selected over all the other adaptive schemes because of its unique features including convergence speed, low computational requirements, and its suitability for processing the data in blocks. The weight adaptation is performed once per block of data instead of per sample of the desired and reference signals. This makes the algorithm very efficient computationally, compared with other LSbased schemes. The algorithm consists of two steps. In the first step, an order update rule is used intrablockwise to successively increase the order of the filter with the exact solution obtained at the $P$ th iteration, where $P$ is the order of the transveral filter. Once the final weight vector is obtained, the filtering is implemented on the data within the whole block in one pass.

\section{RESUltS AND DisCUSSION}

The TDE scheme was examined on two data sets for simulated and real backscattered signals. The goal of the first case was to test the effectiveness of this scheme in resolving multispecular returns with known locations and amplitudes. Different conditions such as low amplitude and closely spaced specular components in the simulated backscattered were considered under different SNR's. The goal of the second experiment was to investigate the performance of the TDE on actual backscattered signals for broad-beam insonification.

\section{A. Results on Synthesized Data}

The synthesized backscattered signal $y(n)$ was generated using the model in (1) with a linear FM incident signal, and $s_{i}(n)=A_{i} \delta(n)$, where $\left\{\Delta_{i}\right\}$ and $\left\{A_{i}\right\}, i \in[1, M]$ represent the $i$ th delay and the associated amplitude for the $i$ th specular component. Six simulated backscattered signals were generated. Table I shows the actual delays and the corresponding amplitudes of each of the components as well as the SNR values. The sub-band or multiresolution decomposition using the DWT was first carried out on both signals for three levels of decomposition using Daubechies (Db6) wavelets [7]. Precautions were taken to avoid edge effects in computing DWT coefficients 


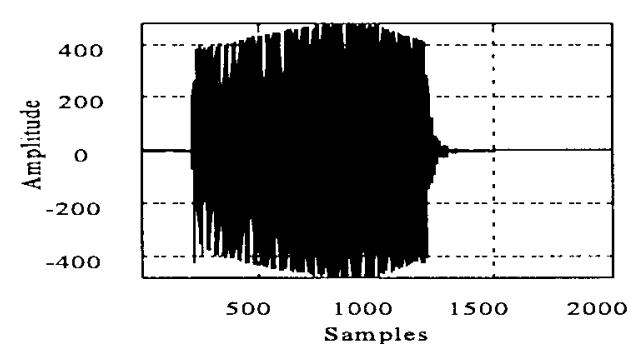

(a)

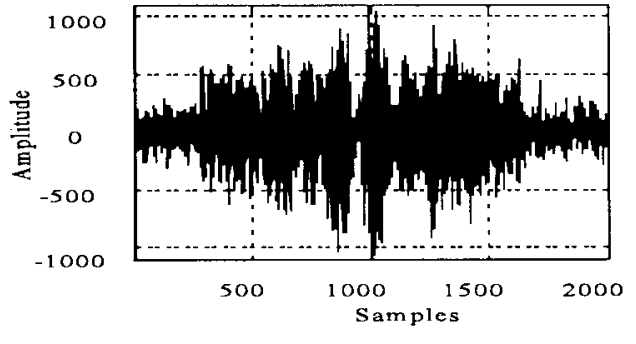

(b)

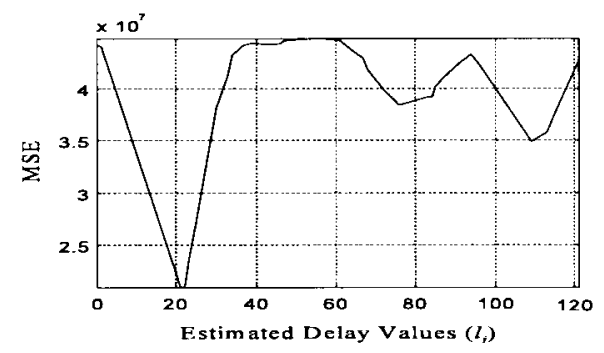

(c)

Fig. 2. (a) Linear FM incident signal. (b) Synthesized backscattered signal for the sixth case of Table I (SNR $=2 \mathrm{~dB}$ ). (c) MSE curve for the sixth case.

for finite length signals by considering symmetric extension of the original signals. An eighth-order adaptive filter was employed in all the cases, and the original BFTF was used for adaptation of the transversal filter weights. The time delay updating steps in the LM algorithm are determined based on the gradient of the cost function according to (10). Table I shows the estimated delay values generated using the TDE scheme for the lowest order approximation in the DWT decomposition. The backscattered signals in cases 1 and 3 contained four components with time separation of more than 100 samples, even though the amplitudes of the third case were small. Case 2 contained components with only 40 samples separation, and case 4 contained three components with more than 100 samples apart, but the first delay was not at zero. For cases 5 and 6, the delays and their associated amplitudes are exactly the same as those in case 4 , but the SNR values are 10 and $2 \mathrm{~dB}$, respectively. Fig. 2(a) and (b) present the incident signal and the simulated backscattered signal corresponding to the sixth case of Table I, respectively.

For the first case, the estimated delay values $l_{i}$ at the second level were $l_{1}=1, l_{2}=36, l_{3}=72$, and $l_{4}=113$ corrected by the lag corresponding to the maximum weight of the BFTF filter, i.e., $q_{1}=1, q_{2}=0, q_{3}=0$, and $q_{4}=6$, respectively. The results were then multiplied by $2^{N}$, i.e., where $N=2$, to provide the estimate $\Delta_{i}$ 's, as given in Table I. The first delay at zero was estimated accurately in this case, whereas the second, third, and fourth delays were estimated with an error of $\leq 2$ samples compared with the actual values at the second level. The inaccuracy in the second delay estimate may be attributed to the fact that the actual delay was not divisible by 4 . For the second and third cases in Table I, it was necessary to use the third level of decomposition since the number of minima did not agree with the number of minima at the second level. The first delay was estimated accurately. The estimates of the third and fourth delays have an inaccuracy of one sample compared with the actual delay values at the third level. This was also true for the delays in case 3 . The third component with the actual delay of 270 was not detected in this case due to its low amplitude. For the fourth case in Table I, the MSE curves at the second and third levels agreed in the number of minima detected. The first and second delays were estimated accurately at 60 and 300 samples at the second level.
For the third delay, the TDE scheme provided an estimated value of 420. The result of this case verifies this important property that the proposed TDE scheme does not require time alignment of the incident and backscattered signals. The fifth and sixth cases in Table I show the robustness of the TDE scheme under low SNR, while the number of specular components was kept fixed. Both cases were generated using the actual delays of the fourth case in Table I but with SNR's of 10 and $2 \mathrm{~dB}$, respectively. Fig. 2(c) presents the MSE curve for the sixth case. This curve shows three well-defined minima providing the estimated delays values of $l_{1}=21, l_{2}=83$, and $l_{3}=109$ at the second level of decomposition with the corresponding lags of $q_{1}=6, q_{2}=6$, and $q_{3}=2$, respectively. These values lead to the time delay estimates given in the last row of Table I. The computation time was measured to be approximately $20 \mathrm{~s}$ for each case.

For comparison purposes, a generalized matched filter in [2] was also implemented on the backscattered signal associated with the last case of Table I. The estimated delays in this case were found to be 61 and 417 . The delay at 300 was not picked up by this method, due to its relatively low amplitude and the high level of noise.

Finally, to show the ability of the adaptive filter in removing the effects of the unknown channel, a synthesized backscattered signal was generated with two components at time lags 0 and 100. These components were then highpass filtered using two fifthorder Butterworth filters with different cutoff frequencies. This first component at zero lag was filtered using a filter that passes less than half of the signal bandwidth, whereas the second component was only slightly filtered. Fig. 3(a) shows this synthesized backscattered signal after noise was added to achieve $\mathrm{SNR}=2 \mathrm{~dB}$. The proposed sub-band TDE method was then applied to this signal. The order of the adaptive filter was five in this case. Fig. 3(b) shows the plot of the MSE curve at the third level of decomposition and for the lowest order approximation sub-band. Using the proposed TDE method, the estimated time delays and the corresponding filter lags were found to be $l_{1}=0, l_{2}=17$, with $q_{1}=0, q_{2}=4$, respectively, leading to the final time delay estimates at 0 and 104. Using the generalized matched filter, the first delay at zero lag was not detected due to the severe distortion in the first component and the fact that the matched filter is not capable of compensating for this distortion. The 


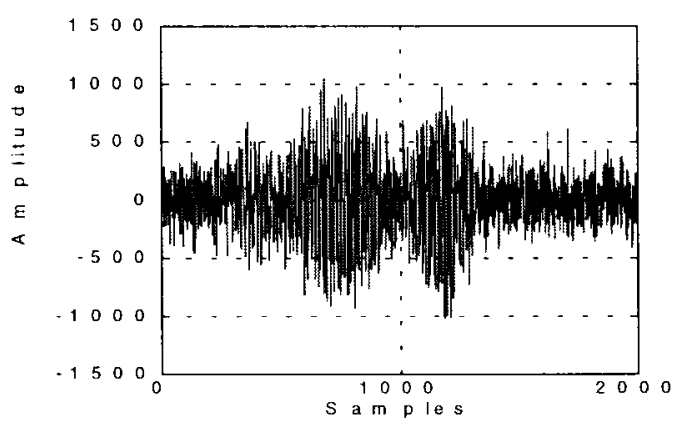

(a)

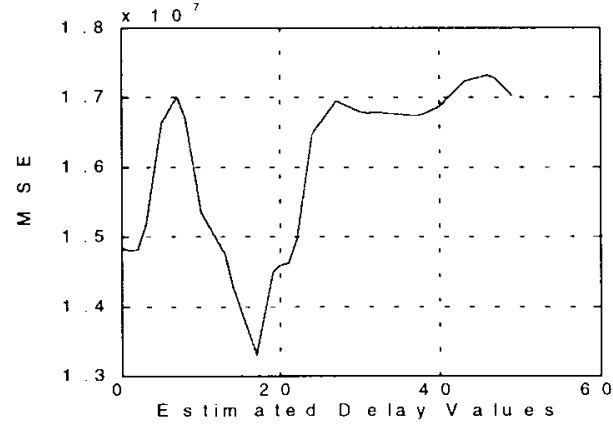

(b)

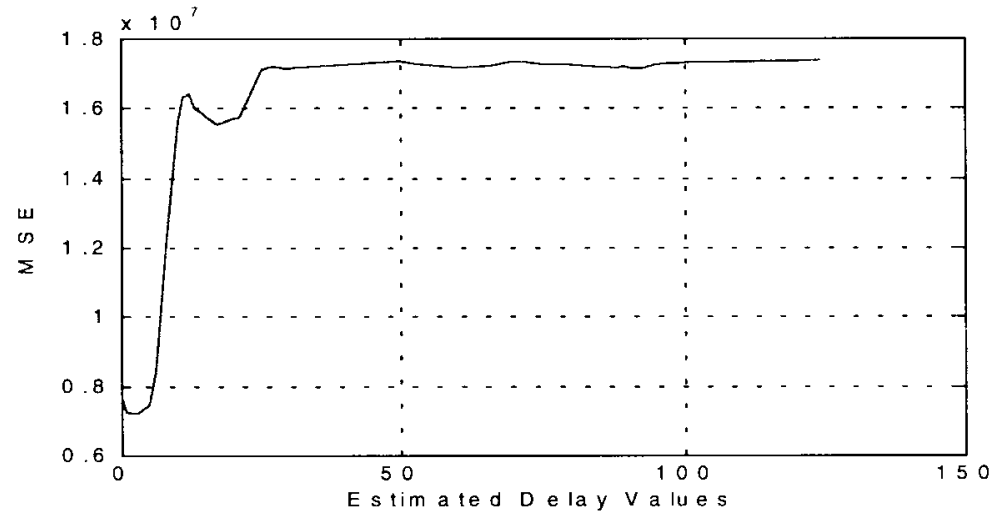

(c)

Fig. 3. (a) Synthesized backscattered signal with highpass filtering. (b) MSE curve for this case. (c) MSE curve for the no-channel effect case.

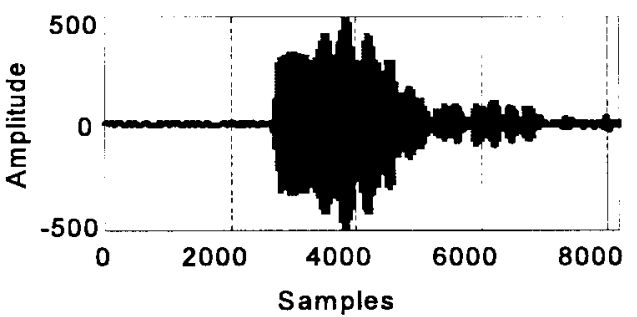

(a)

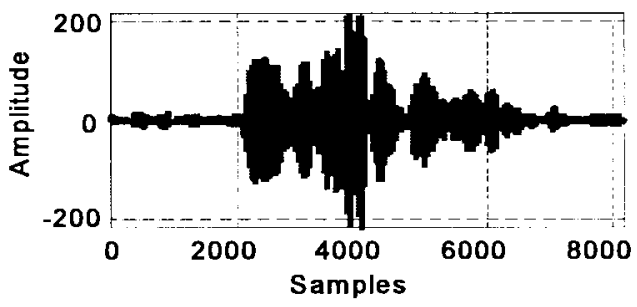

(c)

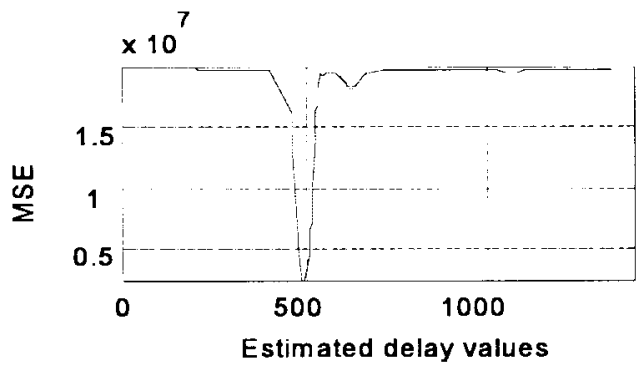

(b)

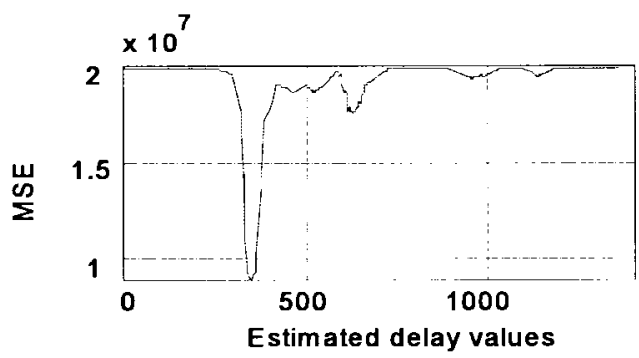

(d)

Fig. 4. (a), (c) Backscattered signals at aspect 0 and $200^{\circ}$.

(b) and (d) MSE curves for aspect angles 0 and $200^{\circ}$, respectively.

estimate of the second time delay was found to be 117. The same experiment was also repeated when the unknown channel effects were absent. Fig. 3(c) shows the plot of the MSE curve at the third level of decomposition for this case. All other conditions were the same as before. The estimated delays using the sub-band TDE method were found to be 0 and 96 . The matched filter method, on the other hand, provided the estimate of the second delay of 110, whereas the first one was missed again.

\section{B. Results on Real Data}

The proposed TDE scheme was also examined on the actual broadbeam acoustic backscattered data. This data set consisted of an incident signal and the backscattered for a submerged elastic target. The target had the form of a tapered, notched cylinder with flattened ends and rivets and an aspect ratio of 4 to 1 . The target was attached to a support mounted on a rotator. The transducer and hydrophone were fixed, and the acoustic backscatter for various target aspect 
angles were measured by pivoting the target lengthwise. Becuase of the broadbeam nature of the incident, which was a wideband linear FM, the presence of multispecular returns is inevitable. Fig. 4(a) and (b) presents the actual backscattered signal at aspect angles of 0 and $200^{\circ}$, respectively. Note that $0^{\circ}$ corresponds to broadside incidence. Fig. 4(c) and (d) shows the corresponding MSE curves obtained at the second level for these cases. Two minima are shown in the MSE curves, indicating two main specular returns with the first one being the more prominent one. The estimated delays were found to be 1964 and 2484 for the $0^{\circ}$ case and 1376 and 2484 for the $200^{\circ}$ case. The changes in the location of the main specular return from the target correspond geometrically to the target rotation. That is, as the target is rotated off broadside $\left(0^{\circ}\right)$, the total round trip travel time from the transducer to the target and target to the hydrophone becomes smaller. The delay corresponding to test facility reverberation, however, remains invariant to target aspect because the transducer and hydrophone are fixed.

\section{Conclusions}

As is evident from the simulation results for several synthesized and real backscattered signals, the proposed TDE scheme provides a promising tool for estimating time delays, particularly in a noisy and cluttered environment. Performing TDE in subbands provides multiple estimates for the time delays, which are generated independently based on the data of each sub-band. This provides a mechanism for validating the estimates of the time delays in different levels/subbands. Additionally, as a result of filtering and decimation processes in each level, the sequences at upper levels are less correlated than the sequences at the lower levels. Consequently, noise and other disturbances will be less prominent in the upper levels than in the original signal domain; moreover, since the signals in the upper levels are less correlated, the estimates of the gradients in the LM algorithm will be less noisy. In addition, due to the decimation process, the signals at upper levels are considerably shorter in size; hence, the operations in each sub-band can be accomplished substantially faster than those in the finest scale. A parallel processor may be used to perform all the sub-band operations simultaneously.

\section{REFERENCES}

[1] M. R. Azimi-Sadjadi, J. Wilbur, and G. Dobeck, "Isolation of resonance in acoustic backscatter from elastic targets using adaptive estimation schemes," IEEE J. Oceanic Eng., vol. 20, pp. 346-353, Oct. 1995.

[2] G. C. Carter, Coherence and Time Delay Estimation. An Applied Tutorial for Research, Development, Test and Evaluation Engineers. New York: IEEE, 1993

[3] A. H. Quazi, "An overview on the time delay estimate in active and passive systems for target localization," IEEE Trans. Acoust., Speech, Signal Processing, vol. ASSP-29, pp. 527-533, June 1981.

[4] A. O. Hero and S. C. Schwartz, "A new generalized cross correlator," IEEE Trans. Acoust., Speech, Signal Processing, vol. ASSP-33, pp. 38-45, Feb. 1985.

[5] B. M. Bell and T. E. Ewart, "Separating multipaths by global optimization of a multidimensional matched filter," IEEE Trans. Acoust., Speech, Signal Processing, vol. ASSP-34, pp. 1029-1037, Oct. 1986.

[6] R. J. Vaccaro, C. S. Ramalingam, and D. W. Tufts, "Least-square timedelay estimation for transient signals in a multipath environment," $J$. Acoust. Soc. Amer., vol. 92, pp. 210-219, July 1992.

[7] I. Daubechies, Ten Lectures on Wavelets. Philadelphia, PA: SIAM, 1992.

[8] M. Vetterli and J. Kovacevic, Wavelets and Sub-band Coding. Englewood Cliffs, NJ: Prentice-Hall, 1995.
[9] J. Dennis and R. Schnabel, "Numerical methods for unconstrained optimization and nonlinear equations." Englewood Cliffs, NJ: PrenticeHall, 1983.

[10] J. M. Cioffi, "The block-processing FTF adaptive algorithm," IEEE Trans. Acoust., Speech, Signal Processing, vol. ASSP-34, pp. 77-90, Feb. 1986.

\section{Rational Sampling Filter Banks Based on IIR Filters}

\author{
Fabrizio Argenti and Enrico Del Re
}

\begin{abstract}
In this correspondence, the problem of splitting the spectrum of a digital signal by using nonuniform infinite impulse response (IIR) filter banks is addressed. Near perfect reconstruction (NPR) is considered. The method uses the modulation of different IIR prototypes. The cancellation of the main aliasing components constrains the prototypes to be dependent on each other. By using this approach, linear-phase prototypes are needed, and noncausal filtering is required. Numerical examples of filter bank design are given, and the computational complexity is compared with the finite impulse response (FIR) case.
\end{abstract}

Index Terms-IIR filter banks, nonuniform filter banks.

\section{INTRODUCTION}

Filter banks [1] are used in several applications in the field of digital signal processing to split the spectrum of a signal. A uniform width subband splitting is usually considered. In some cases, however, a nonuniform splitting can be preferable. Designing nonuniform filter banks is more complex than uniform ones. The problem is addressed, for example, in [2]-[7], where examples of FIR filter bank design are shown. Here, we investigate the effectiveness of using cosinemodulated IIR filters to design nonuniform NPR filter banks. The method is an extension to the IIR case of results presented in [8]. Cosine modulation is widely used to design NPR or PR FIR filter banks [9], and recently, uniform PR IIR cosine-modulated filter banks have been proposed in [10]. In most of the cases, the modulation of a linear-phase prototype is assumed. Since we will exploit some results based on the linear-phase property of the prototypes, we will impose this constraint on the IIR case as well so that we will refer, in this study, to noncausal filter banks. The application of noncausal filter banks has been discussed in some recent papers, such as [11] and [12]. Some numerical examples of filter bank design are shown, and the computational complexity is compared with the FIR case [8].

\section{PSeudo-QMF Rational SAmpling Filter BANKS}

Consider the scheme shown in Fig. 1, where analysis/synthesis rational sampling filter banks are shown. In the $m$ th branch, a fraction of the input spectrum equal to $R_{m} / M_{m}$ is extracted (we will consider maximally decimated filter banks, i.e., $\left.\Sigma_{m} R_{m} / M_{m}=1\right)$. The

Manuscript received February 17, 1997; revised February 25, 1998. This work was supported by Italian MURST. The associate editor coordinating the review of this paper and approving it for publication was Dr. S. Mallat.

The authors are with the Dipartimento di Ingegneria Elettronica, Universitá di Firenze, Firenze, Italy.

Publisher Item Identifier S 1053-587X(98)08685-1. 